\title{
Caracterização da Micromobilidade em Redes Sem Fio Infraestruturadas pela Variação da Relação Sinal-Ruído
}

\author{
Kerlla Souza Luz ${ }^{1}$, Priscila Solís ${ }^{1}$, Henrique Garcia ${ }^{1}$, Mylène C. Q. Farias ${ }^{2}$ \\ ${ }^{1}$ Departamento de Ciência da Computação, ${ }^{2}$ Departamento de Engenharia Elétrica - \\ Universidade de Brasília (UNB) \\ Caixa Postal 4466 - 70.910-900 - Brasília - DF - Brasil \\ kesll@yahoo.com, pris@cic.unb.br, henriquegarcia@gmail.com, \\ mylenedene.unb.br
}

\begin{abstract}
This paper evaluates the viability of using the SNR (signal-noise relation) variation in infrastructured IEEE 802.11 networks for micro-mobility characterization. Using measures that verify the SNR variation in stations with micro-mobility, the paper presents an algorithm to allow a station to make a micro-mobility self-identification procedure and then, may be able to activate a mechanism to diminish management and control frames that verify continuously the connection with the AP (Access Point). The experimental results verify that with several degrees of nodes movement is possible to identify the micro-mobility based in two parameters: the SNR variation and the prevalence with the AP.
\end{abstract}

Resumo. Este trabalho avalia a viabilidade de utilização da variação da SNR (relação sinal-ruído) em redes infraestruturadas IEEE 802.11 para caracterização de micromobilidade. A partir de medidas que verificam a variação de SNR em estações com baixa mobilidade é apresentado um algoritmo para que uma estação possa se auto-identificar com micromobilidade e posteriormente, ativar um mecanismo para diminuir os quadros de gerenciamento e controle que tenham como objetivo apenas a verificação contínua da conexão com o ponto de acesso (AP). Os resultados experimentais permitem observar que com diversos graus de movimentação dos nós é possível identificar a micromobilidade com base em dois parâmetros: a variação da SNR e a prevalência com o AP.

\section{Introdução}

Desde a sua publicação, a camada de enlace da família de protocolos IEEE 802.11 preserva controles que utilizam a premissa de que os usuários têm mobilidade homogênea. Hoje em dia, com os inúmeros cenários de operação das redes 802.11, não é incomum se deparar em ambientes em que a rede conectada mediante meios guiados venha a ser substituída ou complementada por ambientes de conexão sem fio. Nestes ambientes, é possível que a maioria dos usuários sejam caracterizados como usuários com mobilidade reduzida, ou seja, as estações após associar-se a uma Service Set Identification (SSID) permanecem conectadas por longos períodos de tempo com no máximo, curtos intervalos de interrupção. 
A comparação das taxas reais alcançadas pelas redes sem fio com as redes cabeadas propicia o uso das últimas em ambientes que precisam um melhor desempenho. Entretanto, o baixo custo de instalação das redes sem fio aliado à flexibilidade de gerenciamento, pode propiciar que quando os usuários nestas redes apresentem um padrão de mobilidade próximo ou equivalente ao de um usuário de rede cabeada, os mecanismos de controle e gerenciamento da mobilidade possam ser reduzidos de forma adaptativa, propiciando uma maior eficiência na transmissão e aumentando consequentemente a vazão do tráfego de dados.

Alguns trabalhos têm identificado o tráfego indesejado em redes sem fio como aquele que se produz a partir de atividades não produtivas [Raghavendra et al. 2010]. Grande parte do foco da análise tem abordado o tráfego malicioso. Entretanto, uma parcela considerável do tráfego indesejado é gerada por aplicações que utilizam periodicamente mecanismos para manter a conectividade com o usuário, mesmo em casos em que isto não é necessário, por exemplo, em uma rede em que os usuários têm pouca ou nenhuma mobilidade.

Um ambiente de redes sem fio com usuários que apresentam baixa mobilidade é o foco deste trabalho. O conceito de alta prevalência é usado para caracterizar aqueles usuários que mesmo com dispositivos de comunicação móveis, possam ser classificados como estacionários ou de micromobilidade. A micromobilidade pode ser caracterizada a partir de um estudo sobre a alta prevalência em redes sem fio infraestruturadas com um modelo com base na variação da SNR (relação sinal-ruído).

Neste trabalho é apresentado um algoritmo para caracterizar a prevalencia de usuários em redes sem fio infraestutruradas, como parte da futura proposta de um protocolo adaptativo nesses ambientes. $\mathrm{Na}$ seção 2 é apresentada uma revisão de trabalhos sobre tráfego em redes IEEE 802.11. Na seção 3 é definido o conceito de micromobilidde para redes infraestruturadas com base na prevalência e o modelo de propagação adotado deste trabalho. $\mathrm{Na}$ seção 4 são apresentados os resultados experimentais e por fim, a seção 5 apresenta as conclusões e trabalhos futuros.

\section{Tráfego nas Redes IEEE 802.11}

As redes sem fio são definidas pela família de padrões IEEE 802.11 que especifica a camada física e a camada de controle de acesso ao meio. Os quadros são agrupados em três tipos: gerenciamento, controle e dados. Os quadros de gerenciamento permitem que as estações estabeleçam e mantenham conexões. Os quadros de controle ajudam a garantir a entrega dos quadros de forma correta.

A maioria das redes locais sem fio implantadas no padrão IEEE 802.11, operam no modo infraestruturado onde, o ponto de acesso central ou Access Point (AP) está presente [Walke 2006]. Embora canais de acesso sejam descentralizados, todo o tráfego da rede é gerenciado e controlado no AP.

Para manter a funcionalidade da camada de enlace, dois tipos de pacotes são extremamente utilizados durante o tempo de associação do nó, a saber: Beacon e Probe, usados nos procedimentos de escaneamento passivo e ativo para a oferta e seleção de cobertura. Entre intervalos regulares o AP pode enviar pacotes Beacon em broadcast a fim de anunciar sua presença e seus parâmetros atuais de conexão. O pacote Probe 
Request é enviado pela estação para o AP solicitando indicadores de cobertura para garantir o enlace com a melhor oferta.

Recente trabalho publicado [Ganji 2013], descreve uma estratégia agressiva para redução da densidade de uso de APs onde, uma vez que os pacotes do tipo Probe captam o sinal dos dispositivos vizinhos e esse sinal ao ser comparado com o sinal atual, não resulta em associação, os próprios APs poderiam se autoadaptarem para que estações que estejam com sinais melhores possam diminuir a solicitação de informações a esses pontos de acesso numa espécie de camuflagem.

Em [Bento et al. 2010] a partir da análise de amostras reais de tráfego foi realizado uma análise dos tipos de quadros na camada de enlace. Neste trabalho o autor reconhece que cada tipo de quadro corresponde a cerca de um terço da quantidade total de quadros trafegados na rede, com uma leve desvantagem para os quadros de dados. A distribuição de quadros para cada tipo e subtipo teve a seguinte divisão: $34,46 \%$ dos quadros de controle, $33,57 \%$ de gerenciamento e $31,95 \%$ de dados.

Pacotes de dados nulos (null frames) são um tipo especial e importante das WLANs (Wireless Local Area Network). A sua maior característica é não carregar dados e seu uso não é especificado no padrão IEEE 802.11 [Gu et al. 2010]. Entretanto, a indústria fabricante desses tipos de equipamentos os usa numa ampla variedade de aplicações, a saber: gerenciamento de energia, varredura de canal e o estado (ativo ou ocioso) da estação, entre outras. Quando usado para manter a associação, os pacotes nulos de uma forma geral notificam o ponto de acesso da existência de estações ociosas. Percebe-se que, o uso desse tipo de pacote é facilitado pelo seu pequeno tamanho e flexibilidade de implementação.

Esse tipo de pacote ainda é usado pela estação móvel para informar ao AP sobre mudanças de estados. Pontos de acessos nunca transmitem pacotes nulos - porém nenhuma especificação do 802.11 os impede de fazê-lo, entretanto, não há razões do seu uso pelo AP [Gast 2005].

A diferenciação do tráfego nas redes sem fio tem uma importância relevante na camada de enlace. Em [Raghavendra et al. 2010] foram estudados padrões do tráfego em que uma parte significativa do tráfego na camada de enlace, que permite os mecanismos que iniciam, mantém e alteram as conexões entre o cliente e o ponto de acesso, parece ser não necessária, principalmente quando existe baixa mobilidade dos usuários. Observa-se que uma grande parcela desse tráfego pode contribuir para o agravamento dos problemas de conexão da camada de enlace em que clientes erroneamente concluem que perderam conexão com o ponto de acesso e reiniciam handoffs, mesmo na ausência de mobilidade.

\section{Conceitos de Prevalência e Persistência em redes sem Fio e o Modelo de Propagação para Ambientes Indoor}

Em [Paxson 1996], o conceito de prevalência foi definido como a probabilidade global de uma determinada rota ser encontrada. Adaptando esse enfoque para o uso em redes locais sem fio, o valor de prevalência é a probabilidade de uma estação sem fio permanecer associada a um determinado ponto de acesso disponível dentro do espaço físico coberto por uma rede local. Dessa forma, este conceito indicará a possibilidade de 
um cliente se associar seguidamente a um mesmo ponto de acesso (AP) tendo em vista uma estrutura de serviço estendida formado por múltiplos APs (ESS). O valor da persistência será entendido como sendo os tempo decorrido do inicio ao fim de cada associação de um nó ao AP. A prevalência é então um indicativo da mobilidade dos clientes. Se um cliente é estacionário - predominantemente não móvel - a prevalência de uma associação entre um nó e o AP será considerada alta, porém, se os valores de prevalência estiverem distribuídos uniformemente pode indicar que o cliente tenha alternado a conexão com outros pontos de acesso vizinhos e se movimentado fisicamente pela área de cobertura. A prevalência $\pi$ do cliente conectado a um determinado AP é definido pela Eq. 1 [Paxson 1996].

$$
\pi=k s / N s
$$

Em (1) ks representa o tempo total que o cliente esteve ativo e $N s$ representa o tempo total de avaliação da amostragem. Utilizando-se (1) para um cenário com baixa mobilidade, espera-se que os clientes repetidas vezes se associem ao mesmo AP ainda que com múltiplos pontos de acessos ao alcance do nó. Para o cálculo da persistência, mede-se o tempo decorrido de conexão entre o momento que o cliente associa-se a um determinado AP até o encerramento da conexão sem interrupções, dado pelo termo ks.

O modelo 802.11 trabalha no sentindo de manter o enlace ativo a qualquer custo, o que na prática faz com que estações móveis e APs troquem pacotes nulos (sem dados) para permanecer conectados e assim, garantir que o cliente permaneça ativo junto ao mesmo AP ao qual estava associado. O valor da persistência é usado para a análise da prevalência e será entendido como sendo intervalos de tempo decorridos do início ao fim de cada associação de um nó ao AP.

Existem vários modelos de predição de intensidade de campo eletromagnético padronizados por comissões internacionais (COST) para redes sem fio, entre eles podese citar: o modelo de Okumura, Hata e Lee [Alencar 2009], porém todos classificados pela literatura para áreas urbanas.

O nível de sinal recebido decresce logaritmicamente com a distância [Vasco C. L. et al., 2013] e dessa forma, o modelo de Perda de Propagação Log-distância foi adotado neste trabalho para caracterizar a propagação indoor, conforme [Jadhavar B.R, Sontakke T.R, 2012][Sulaiman A.I, Hussein A.M, 2012], definido pela Equação 3, em que $P L$ é a perda de propagação entre as distâncias $d$ e $d_{0}, d$ é a distância do transmissor ao ponto medido, $d_{0}$ é uma distância de referência próxima ao transmissor e $n$ é o expoente da perda de propagação.

$$
P L(d)=P L\left(d_{0}\right)+10 n \log \left(d / d_{0}\right)
$$

O ambiente interno causa perdas mais acentuadas do que na condição de espaço livre. Este modelo de normalização logarítmica onde a perda de propagação é caracterizada por um fator de atenuação, que no caso, é o expoente de distância $n$ é um modelo não dependente da frequência podendo ser utilizado em várias faixas de transmissão, e o valor de $n$ contém intrinsecamente o efeito de todos os mecanismos de propagação [Vasco C. L. et al., 2013]. O valor de $n$ leva em consideração a existência de ambientes diferenciados para o percurso do sinal e se adapta tanto para ambientes internos ou externos. Podendo variar entre 2 a 5 para ambientes externos e de 4 a 6 para ambientes internos [Sulaiman A.I, Hussein A.M, 2012]. 


\section{Análise Experimental}

\subsection{Avaliação da SNR em estações com micromobilidade}

Com o objetivo de comprovar a baixa variação dos valores de SNR de nós qualificados como estacionários ou micromobilidade, foram coletadas medidas reais no cenário descrito na Figura 1. As medidas foram conduzidas a partir da observação e análise de 15 nós sem fio, 1 ponto acesso da marca Cisco e 1 adaptador USB sem fio 54Mbps. Todos os dispositivos operaram a versão g do protocolo IEEE 802.11, na frequência de $2.4 \mathrm{GHz}$ e transmissão típica de energia de $18 \mathrm{dBm}$. Os nós foram alocados ao longo de três laboratórios do Centro de Ciência da Computação (CIC) no Campus Darci Ribeiro na Universidade de Brasília (UNB).

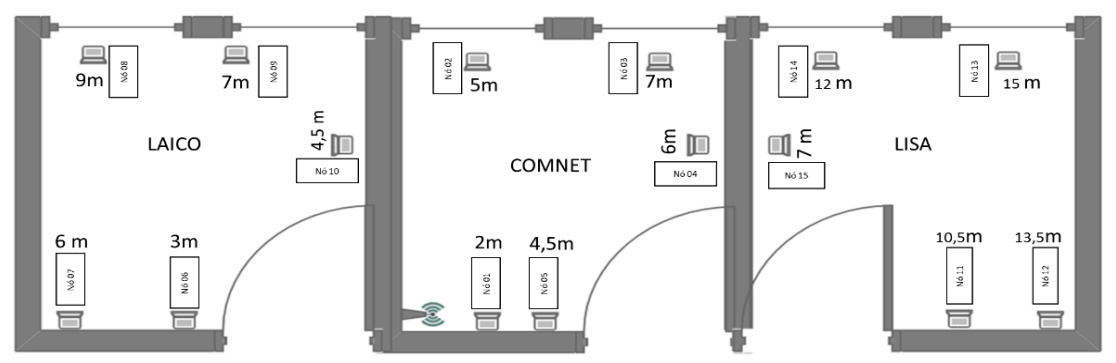

Figura 1. Ambiente Real de Medidas

Durante os testes, cada host autenticava-se ao SSID do AP localizado no ambiente COMNET. Foram registrados para cada nó os valores de potência, ruído e SNR. Por cada nó foram realizadas 10 medidas diferentes por intervalo e foi calculada a média de cada bloco. Os testes foram realizados durante um mês em horários alternados entre os turnos manhã, tarde e noite. Os testes foram operados sob canal 9, com outros 14 pontos de acesso disponíveis na mesma área de cobertura.

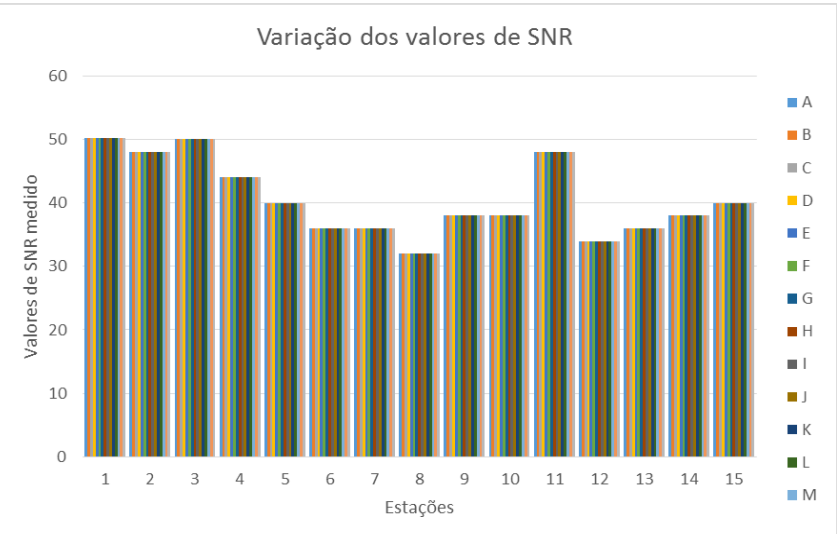

Figura 2. Valores de SNR para cada nó em diversas medidas

A figura 2, representa a relação entre os valores do sinal-ruído para a medida da SNR. Observa-se que para todos os nós medidos há a estabilidade do valor de SNR, uma vez que foram caracterizados como estacionários ou micromobilidade (sofreu pequenas movimentações como por exemplo, de uma mesa para outra próxima). 


\subsection{Algoritmo para caracterização da micromobilidade}

Foi implementado um algoritmo para caracterização da mobilidade das estações. O cenário foi definido como um conjunto de usuários com mobilidade variada, em uma área coberta por um ponto de acesso com raio de 20 metros formado por 105 estações de trabalho (móveis e fixas). Observe-se que o número de estações nesse raio de cobertura caracteriza uma alta densidade de usuários. As premissas utilizadas na simulação foram as seguintes:

A) Utilização do modelo Log-distância para a propagação do sinal, descrito na Equação 3. Na implementação do algoritmo, $n$ considerou-se igual a 1 metro (m), ou seja, a distância do transmissor ao ponto medido mais próximo da antena e $\operatorname{PL}\left(d_{0}\right)$ a média da perda de propagação dos pontos medidos a $1 \mathrm{~m}$.

B) Os parâmetros mostrados na tabela 2 foram utilizados conforme referencia do manual do AP do tipo Roteador wireless G broadband [Cisco Systems 2011] na intenção de englobar o padrão da grande maioria de roteadores disponíveis no mercado. Estes valores podem ser mudados durante a simulação para possibilitar a análise de muitos tipos de situações nas quais o sistema possa ser submetido.

C) Para o cálculo da prevalência, nesta versão do algoritmo proposto, como limitação do modelo de simulação, o algoritmo descrito e implementado considera o cálculo da prevalência constante em função de que na área coberta simulada há apenas um único AP representado por $(\pi=1)$ onde, o valor 1 significa alta prevalência, ou seja, as estações podem ter baixa mobilidade na área coberta pelo AP mas não deverão sair desta área de cobertura.

D) O SNR é utilizado como medida do grau de movimentação a partir do uso de modelos de perda de caminho para estimar o nível do sinal recebido como uma função da distância. O algoritmo de adaptação irá armazenar os valores da SNR a cada verificação para indicar ao final de cada três verificações a adaptação ou não da estação.

O algoritmo 1 detalha a implementação do processo anteriormente descrito. Para mudar de estado, a estação deverá ter mobilidade reduzida, a qual será medida pelos valores de SNR trocados entre o nó, o AP e o da prevalência. Porém, numa rede com mais pontos de acessos se levaria em consideração o tempo de conexão com cada um destes pontos de acesso (persistência) para então decidir pela adaptação ou não. É de suma importância ressaltar que o processo de identificação da micromobilidade é executado pela própria estação, o que de certa forma desonera o AP em ter que se preocupar com este processo. Porém, idealiza-se que uma vez adaptado em um sistema real, o AP deverá ser informado para então, minimizar o envio de pacotes apontados como desnecessários.

\subsection{Resultados}

Inicialmente foi necessário identificar os parâmetros descritos nas tabelas 02 e 03 para a configuração adequada do algoritmo. Também foram feitas convenções decorrentes do estabelecimento de parâmetros relacionados aos valores de tempo, tratados como unidade de tempo (ut) e velocidade como unidade de velocidade (uv). A Tabela 1 resume os símbolos, valores e unidades utilizados na simulação. 
No inicio da simulação, todos os nós associados ao mesmo AP começam no estado normal, sem nenhum mecanismo de adaptação. Na dinâmica de simulação, após três sequiências de testes em que os valores de SNR são observados, o algoritmo irá comparar aos valores de referência e então decidir pela adaptação quando necessário. Caso o nó já esteja adaptado e a comparação dos valores permanecer dentro da referência o nó permanecerá nesse estado, caso contrário, deverá mudar de estado. Após perder o estado de ativação do mecanismo, reinicia-se o processo de verificação para todo o fluxo necessário à ativacão.

Algoritmo 1. Procedimento para adaptação da estação baseado na variação de SNR

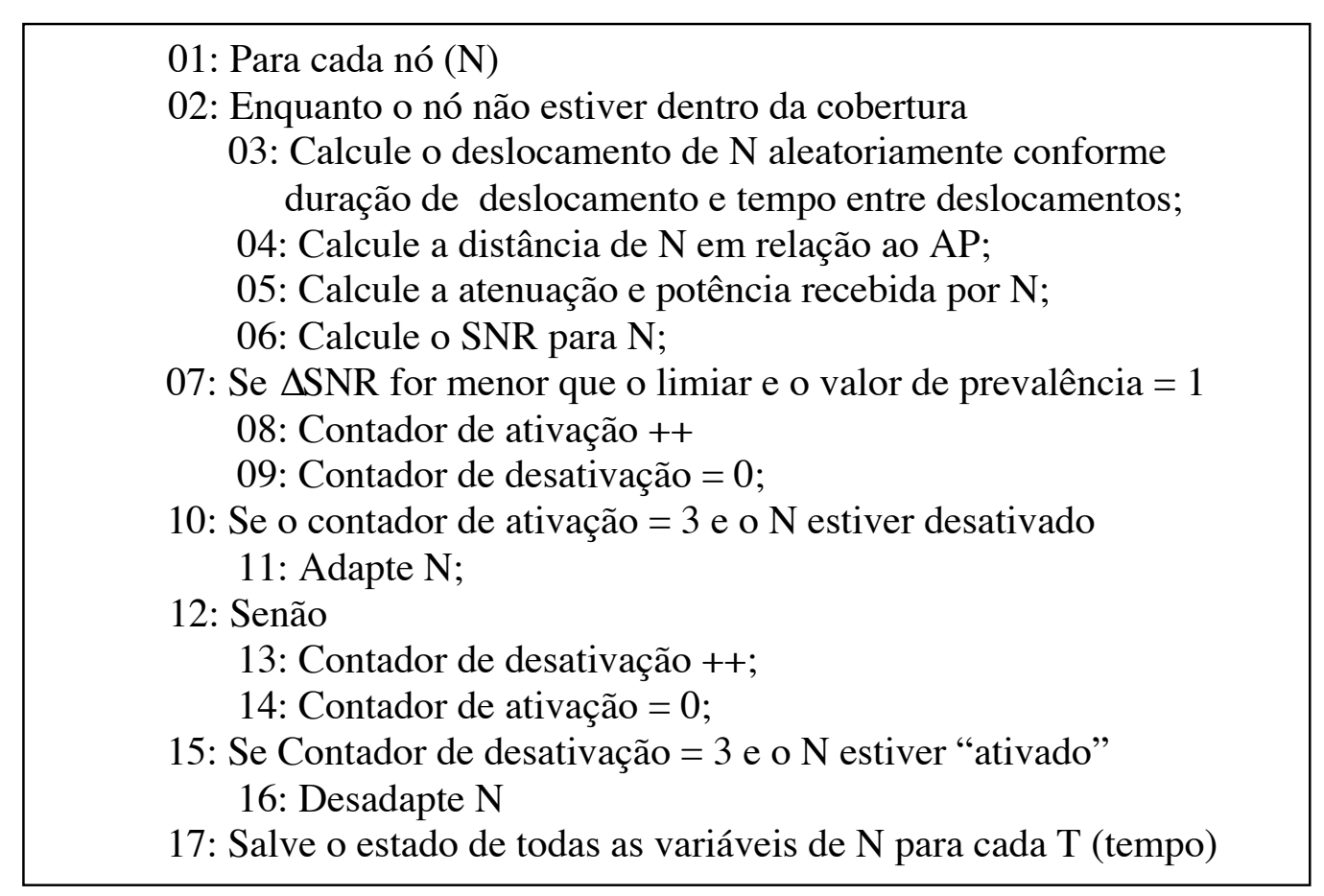

Tabela 1. Parâmetros da Simulação

\begin{tabular}{|c|c|c|c|}
\hline PARÂMETROS & SímBOLOS & VALORES & UNID. \\
\hline Frequência de Operação & $\mathrm{F}$ & 2.4 & $\mathrm{GHz}$ \\
\hline Potência de Transmissão (cliente) & $\mathrm{PMt}$ & 13 & $\mathrm{dBm}$ \\
\hline Ganho da antena do AP & $\mathrm{Gt}$ & 2 & $\mathrm{dBi}$ \\
\hline Ganho da antena da estação (cliente) & $\mathrm{Gr}$ & -1 & $\mathrm{dBi}$ \\
\hline
\end{tabular}

Tabela 2. Parâmetros descritivos para o cenário proposto

\begin{tabular}{|c|c|c|}
\hline PARÂMETROS & VALORES & UNIDADE \\
\hline Raio de cobertura do AP & 20 & $\mathrm{~m}$ \\
\hline Velocidade de deslocamento do nó & 0 a 5 & uv \\
\hline Duração do deslocamento & 1 a 6 & ut \\
\hline Tempo entre deslocamento & 100 & ut \\
\hline Numero de teste antes da adaptação & 3 & ut \\
\hline Tempo entre os testes & 1 & ut \\
\hline Tempo total da simulação & 600 & ut \\
\hline Posição inicial da estação & 5 & x e y \\
\hline Numero de estações por simulação & 105 & nó \\
\hline
\end{tabular}


Conforme mostrado na Tabela 2, foi considerado raio de cobertura do AP de 20 metros, velocidade de deslocamento do nó de 0 a 6 uv, duração do deslocamento de 1 a 6 ut, tempo entre o deslocamento de 100 ut, número de teste antes da adaptação igual a 3 e tempo entre os testes de 1 ut. Durante a simulação outros valores foram utilizados, porém, não houve alterações significativas nas médias de ativação do mecanismo. Dessa forma, esses dados representam valores médios dentro da escala de valores testados. Os parâmetros utilizados foram: frequência de operação $(\mathrm{F})$ com valores de $2,4 \mathrm{GHz}$, a potência de transmissão do cliente $(\mathrm{Pt})$ de $20 \mathrm{dBm}$ potência do ruído de $-200 \mathrm{dBm}$.

A Tabela 3 mostra o resultado da simulação com os resultados classificados por faixas de variações da SNR. Percebe-se que mesmo as estações com macromobilidade puderam ser identificadas pois o algoritmo previa paradas entre as movimentações, o que permitia que os nós se movimentassem ao longo da área de cobertura.

As figuras 3 e 4 ilustram a movimentação e a ativação das estações simuladas pelo algoritmo. Os eixos $x$ e $y$ são componentes da posição do nó em relação ao AP, tendo em vista a sua localização na posição $(0,0)$ e cobertura máxima de 20 metros. A oscilação nos eixos $y$, várias linhas em um, significa adaptado e sete, significa desadaptado. Nos eixos $x$ têm-se o tempo de simulação. As Figuras 3.a e 3.b representam cinco estações com nenhuma mobilidade (estacionárias) derivando em gráficos sem oscilações, permanecendo sempre no estado de adaptação permanentemente (b).

As Figuras 4.a e 4.b representam estações com alta mobilidade (macromobilidade). Na figura (a) pode-se observar o deslocamento total das mesmas dentro da área coberta. $\mathrm{Na}$ imagem (b) observa-se que alguns nós obtiveram períodos de ativação (linhas oscilando na vertical) ou nenhuma (linha na horizontal) em função da variação do valor de SNR. Dessa forma, conclui-se que as estações com maior movimentação experimentaram uma maior variação nos valores de SNR e dessa forma, não poderiam em uma situação real ativarem o mecanismo de adaptação.

Os resultados anteriores mostram que com base nos parâmetros de prevalência e variação da relação sinal-ruído é possível identificar o momento em que uma estação poderia iniciar uma versão adaptativa do protocolo IEEE 802.11. Da mesma forma, o algoritmo proposto permite identificar o momento em que a estação deve sair dessa dinâmica adaptativo.
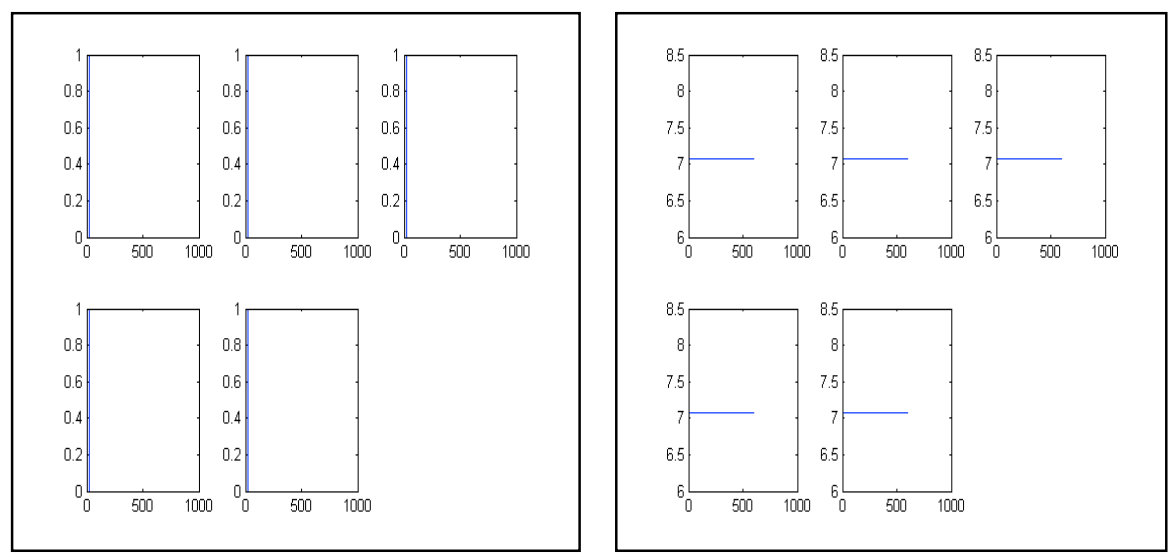

Figura 3. Movimentação(a) e Ativação(b) de estações estacionárias 
Tabela 3. Resultados da simulação

\begin{tabular}{|l|l|l|l|}
\hline \multicolumn{1}{|c|}{$\Delta$ SNR } & \% ADAPTAÇÃO & \multicolumn{1}{c|}{$\begin{array}{c}\text { MÉDIA } \\
\text { MOVIMENTAÇÃO }\end{array}$} & \multicolumn{1}{c|}{ Classificação } \\
\hline 0 a 0,09 & $83 \%$ & 39,08 & Estacionárias \\
\hline 0,10 a 0,19 & $67 \%$ & 303,51 & Micromobilidade \\
\hline 0,20 a 0,29 & $61 \%$ & 721,38 & Micromobilidade \\
\hline 0,30 a 0,39 & $51 \%$ & 1728,18 & Macromobilidade \\
\hline 0,40 a 0,49 & $30 \%$ & 3236,16 & Macromobilidade \\
\hline
\end{tabular}
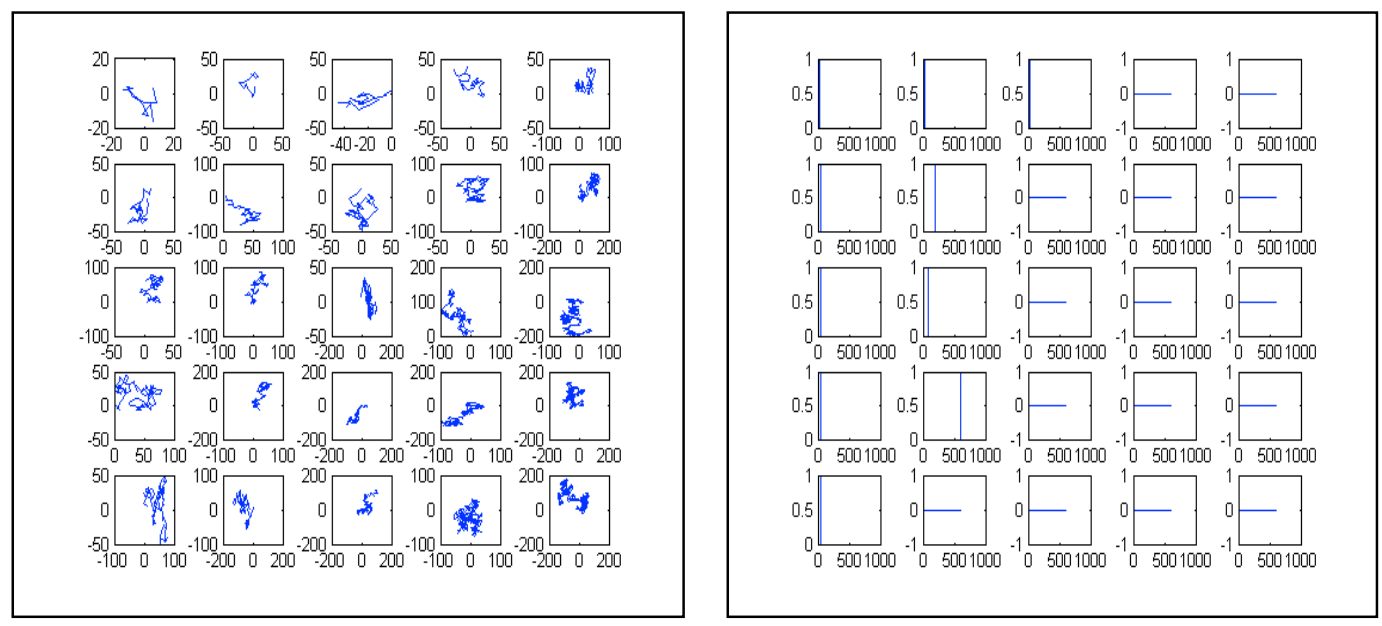

Fig. 4. Movimentação(a) e Ativação(b) de estações com macromobilidade

\section{Conclusões}

Neste artigo foi proposto um algoritmo para implementar um mecanismo de tráfego adaptativo para o protocolo IEEE 802.11 no caso de usuários com micromobilidade. A proposta teve como foco usuários suscetíveis à adaptação do tráfego de pacotes com o intuito primordial de minimizar o nível de congestionamento em redes com alta densidade e aumentar a eficiência na transmissão.

O estudo se baseia em trabalhar com dois parâmetros: a prevalência e a variação da relação sinal-ruído, calculada em cada nó móvel. Os resultados experimentais mostram que a verificação destes valores em intervalos regulares permitem identificar o momento em que uma estação poderia iniciar uma versão adaptativa do protocolo IEEE 802.11. Da mesma forma, o algoritmo proposto permite identificar o momento em que a estação deve sair dessa dinâmica adaptativa. Os resultados de um ajuste no protocolo permitiriam reduzir o volume de tráfego de gerenciamento e controle na rede, aumentando por sua vez o volume de tráfego de dados, com uma melhora de desempenho na rede. 
Como trabalho futuro, deverá ser implementado um conjunto de alterações no protocolo da família IEEE 802.11 com base nesta proposta de mecanismo de adaptação para estações com micromobilidade em redes infra-estruturadas.

\section{Referências}

Alencar, M. S. (2010), Ondas eletromagneticas e teoria de antenas, Érica.

Bento, T. F. Barreto, P. S. (2010) "Um Estudo da composição do tráfego Autossimilar na Camada de Enlace para Avaliação do Desempenho em Redes sem fio", XVII Workshop de Gerência e Operação de Redes e Serviços (Anais) ISSN: 2177-496X.

Cisco Systems (2011), User Guide Wireless G Broadband Linksys.

Ganji, F., Budzisz, L., Wolisz, A., (2013) "Assessment of the Power Saving Potential in Dense Enterprise WLAN", IEEE Annual International Symposium on Personal, Indoor anda Mobile Radio Communications (Proceddings PIMRC), pág. 28502855 .

Gast, M.S. (2005), 802.11 wireless networks: the definitive guide. O Reilly Media, 2nd edition.

Gu, W., Yang, Z., Xuan, D., Jia, W., Que, Can., (2010) "Null Data Frame: A DoubleEdged Sword in IEEE 802.11 WLANs", IEEE Transactions on Parallel and Distributed Systems, VOL. 21, NO. 7.

Jadhavar B.R, Sontakke T.R., "2.4 GHz Propagation Prediction Models for Indoor Wireless Communications Within Building", International Journal of Soft Computing and Engineering (IJSCE), ISSN: 2278-3075, Volume - 2, Issue - 3, July 2012.

Li, Y., Papagiannaki, D., Sheth, A., (2011) "Uplink Traffic Control in Home 802.11 Wireless Networks", ACM SIGCOMM Workshop on Home Networks (Proceddings), ISBN: 978-1-4503-0798-7.

Paxson, V. (1996), "End to End Routing Behavior in the Internet," em SIGCOMM 1996, University of Berkeley.

Raghavendra, R., Elizabeth. M. B., Papagiannaki, K., and Almeroth, K.C., (2010) "Unwanted Link Layer Traffic in Large IEEE 802.11 Wireless Networks", IEEE Transactions on Mobile Computing, VOL. 9, N. 9.

Sulaiman A.I, Hussein A.M , "A Modified Multi-wall Wave Propagation Model for Concrete Based Building Structure" International Conference on Computer and Communication Enginnering (ICCCE 2012).

Vasco C. L. et al., "Ensaios de Transmissão e Recepção de TV Digital Terrestre na Região Metropolitana de Curitiba", Revista de Radiodifusão. Volume-3, nº 03. 2013.

Walke, B. H., (2006), IEEE 802 Wireless Systems, Jonh Wiley. 